\title{
Tyrosine kinase inhibitors targeting FLT3 in the treatment of acute myeloid leukemia
}

\author{
Yun Chen ${ }^{1}$, Yihang Pan ${ }^{2}$, Yao Guo ${ }^{1}$, Wanke Zhao ${ }^{1}$, Wanting Tina Ho ${ }^{1}$, Jianlong Wang ${ }^{3}$, Mingjiang Xu', \\ Feng-Chun Yang ${ }^{4}$, Zhizhuang Joe Zhao ${ }^{1}$ \\ ${ }^{1}$ Department of Pathology, University of Oklahoma Health Sciences Center, Oklahoma City, OK, USA; ${ }^{2}$ The Seventh Affiliated Hospital of Sun Yat- \\ sen University, Shenzhen 518107, China; ${ }^{3}$ Department of Cell, Developmental and Regenerative Biology, Black Family Stem Cell Institute, Icahn \\ School of Medicine at Mount Sinai, New York, NY, USA; ${ }^{4}$ Sylvester Comprehensive Cancer Center, Department of Biochemistry and Molecular \\ Biology, University of Miami Miller School of Medicine, Miami, FL, USA \\ Contributions: (I) Conception and design: All authors; (II) Administrative support: None; (III) Provision of study material or patients: None; (IV) \\ Collection and assembly of data: All authors; (V) Data analysis and interpretation: All authors; (VI) Manuscript writing: All authors; (VII) Final \\ approval of manuscript: All authors. \\ Correspondence to: Zhizhuang Joe Zhao. Department of Pathology, University of Oklahoma Health Sciences Center, Oklahoma City, Oklahoma \\ 73104, USA. Email: joe-zhao@ouhsc.edu.
}

\begin{abstract}
Acute myeloid leukemia (AML) is a cancer of the myeloid lineage of blood cells. Although significant progress has been made in treating many types of cancers during recent years, AML remains a deadly disease with survival rate lagging behind other blood cancers. A combination of toxic chemotherapies has been the standard AML treatment for more than 40 years. With intensive efforts to define the pathogenesis of AML, novel therapeutic drugs targeting key molecular defects in AML are being developed. Mutated in nearly 30\% of AML, FMS-like tyrosine kinase 3 (FLT3) represents one of the most attractive targets. FLT3 mutants resulted from either internal tandem duplication (ITD) or point mutations possess enhanced kinase activity and cause constitutive activation of signaling. To date, several small molecule inhibitors of FLT3 have been developed but their clinical efficacy is limited due to a lack of potency and the generation of drug resistance. Therefore, next-generation FLT3 inhibitors overcoming these limitations are urgently in need. This review focuses on the pathological role of mutant FLT3 in the development of AML, the current status of FLT3 inhibitor development, and mechanisms underlining the development of resistance to existing FLT3 inhibitors.
\end{abstract}

Keywords: Leukemia; FMS-like tyrosine kinase 3 (FLT3); tyrosine kinase inhibitors (TKIs)

Received: 16 March 2017; Accepted: 25 April 2017; Published: 02 June 2017.

doi: $10.21037 /$ sci.2017.05.04

View this article at: http://dx.doi.org/ 10.21037/sci.2017.05.04

\section{Acute myeloid leukemia (AML) overview}

AML is the most frequent form of leukemia, representing approximately $33 \%$ of all leukemia cases in the United States (1). The incidence rate of AML increases with age, and the medium age at diagnosis is 67 years. In 2016, there are 19,950 estimated new cases and 10,430 deaths as a result of the disease (2). A major clinical feature of AML is the presence of myeloblasts in the peripheral blood. These cells infiltrate multiple organs including lung and brain thereby leading to functional abnormalities and even fatality in some cases (3). Anemia and thrombocytopenia are also common features of AML, which lead to weakness, pallor, palpitations, fatigue and various bleeding symptoms (4). Cytochemical and phagocytotic defects in mature myeloid cells can also appear in AML patients, although major infections are uncommon since their absolute counts are usually stable before chemotherapy (5).

Some signs and symptoms related to anemia, neutropenia, and thrombocytopenia may suggest that a 


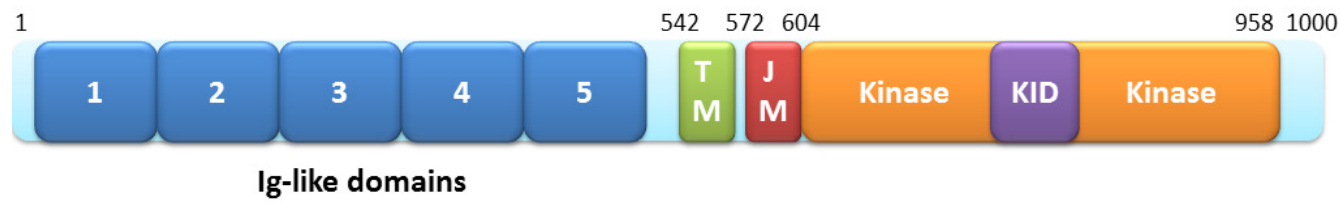

Figure 1 The domain structure of FLT3. The extracellular domain contains five Ig-like domains. Following the transmembrane domain (TM), the intracellular domain contains the juxtamembrane domain (JM) and the kinase domain that is divided into two parts by the kinase insert domain (KID).

person could have AML (6), but various laboratory tests are required to confirm the diagnosis. Having at least 20\% blasts in the marrow is usually required for a diagnosis of acute leukemia (3). A diagnosis can also be made if the blasts are found to have an AML-specific chromosomal abnormality even if blast contents are less than $20 \%$. In most cases, the diagnosis of AML can be achieved by the morphological assessment of peripheral blood and bone marrow samples. But sometimes, especially in the poorly differentiated acute leukemia, cell numbers and morphology may not be enough to distinguish AML from acute lymphoid leukemia (ALL). In these cases, additional tests including cytochemical stains (7) and multi-parametric flow cytometry (8) are required to reach a clear diagnosis.

The therapeutic decision for AML treatment is between the standard and the investigational therapies. Standard therapy follows the so-called " $3+7$ " induction protocol that most often includes three days of idarubicin or daunorubicin and seven days of cytarabine (9). These treatment combinations have been the mainstay of AML treatment for more than 40 years. Investigational therapies include anti-CD33 agents, tyrosine kinase inhibitors (TKIs), hypomethylating agents, and immunotherapies (10). Considering the investigational therapies are under clinical trials and their results are unclear, the standard therapies are usually applied first especially when the successful rate is predicted to be high.

\section{The role of FLT3 in hematopoiesis and AML}

The human FLT3 gene is located on chromosome 13q12 (11). The encoded protein FLT3 belongs to a type III receptor tyrosine kinase whose family members include KIT, FMS and PDGF-receptor (12). The structure of FLT3 consists of five immunoglobulin-like domains in the extracellular region, a juxtamembrane (JM) domain, a tyrosine kinase (TK) domain separated by a kinase insert and a C-terminal domain in the intracellular region (12)
(Figure 1). Human FLT3 have two forms: a $158-160 \mathrm{kDa}$ glycosylated form and a 130-143 kDa non-glycosylated form $(13,14)$. Under normal conditions, FLT3 is only expressed in $\mathrm{CD}_{3} 4^{+}$hematopoietic stem/progenitor cells and play a well-defined role in the development of hematopoietic stem cells, dendritic cell progenitors, B-cell progenitors, and natural killer cells (15). Knockout of FLT3 in mice led to deficiencies in B-cell progenitors, and subsequent transplantation studies further revealed defects in T cells and myeloid cells (16). In colony-forming assays with human CD $34^{+}$bone marrow cells, FLT3 high expressers gave rise to colony-forming unit granulocytemonocyte (CFU-GM) colonies, while FLT3 low expressers produced erythroid colonies (17).

In the inactive state, FLT3 stays in the monomeric form on the cell membrane. Upon stimulation with FLT3 ligand (FLT3L), FLT3 dimerizes and trans-phosphorylates exposed tyrosine residues (18), which results in the activation of multiple downstream signaling pathways $(19,20)$. In this process, a series of signaling and adaptor proteins are involved, including Janus kinase (JAK), signal transducer and activator of transcription (STAT), extracellular-signal regulated kinase (ERK1/2), phosphatidylinositol 3 kinase (PI3K), Src-homology 2 containing inositol phosphatase (SHIP), phospholipase $\mathrm{C} \gamma$ (PLC- $\gamma$ ), CBL, growth factor receptor-bound protein 2 (Grb2), SHC, Src-homology 2 containing protein tyrosine phosphate (SHP-2). Activation of this signaling events leads to the increased cell proliferation, inhibition of apoptosis, and induction of differentiation (Figure 2) (21-23).

Although the expression of FLT3 is limited to the CD34positive population in the normal human bone marrow, its aberrant expression can be found in nearly all cases of primary cells from AML, pre-B ALL, T-cell ALL, blast phase CML, and CLL patients (14). It is also expressed in most pre-B ALL and AML cell lines (24). Co-expression of FLT3 and FLT3L was found in $~ 36 \%$ (40 out of 110) of human leukemia cell lines, indicating autocrine signaling in 


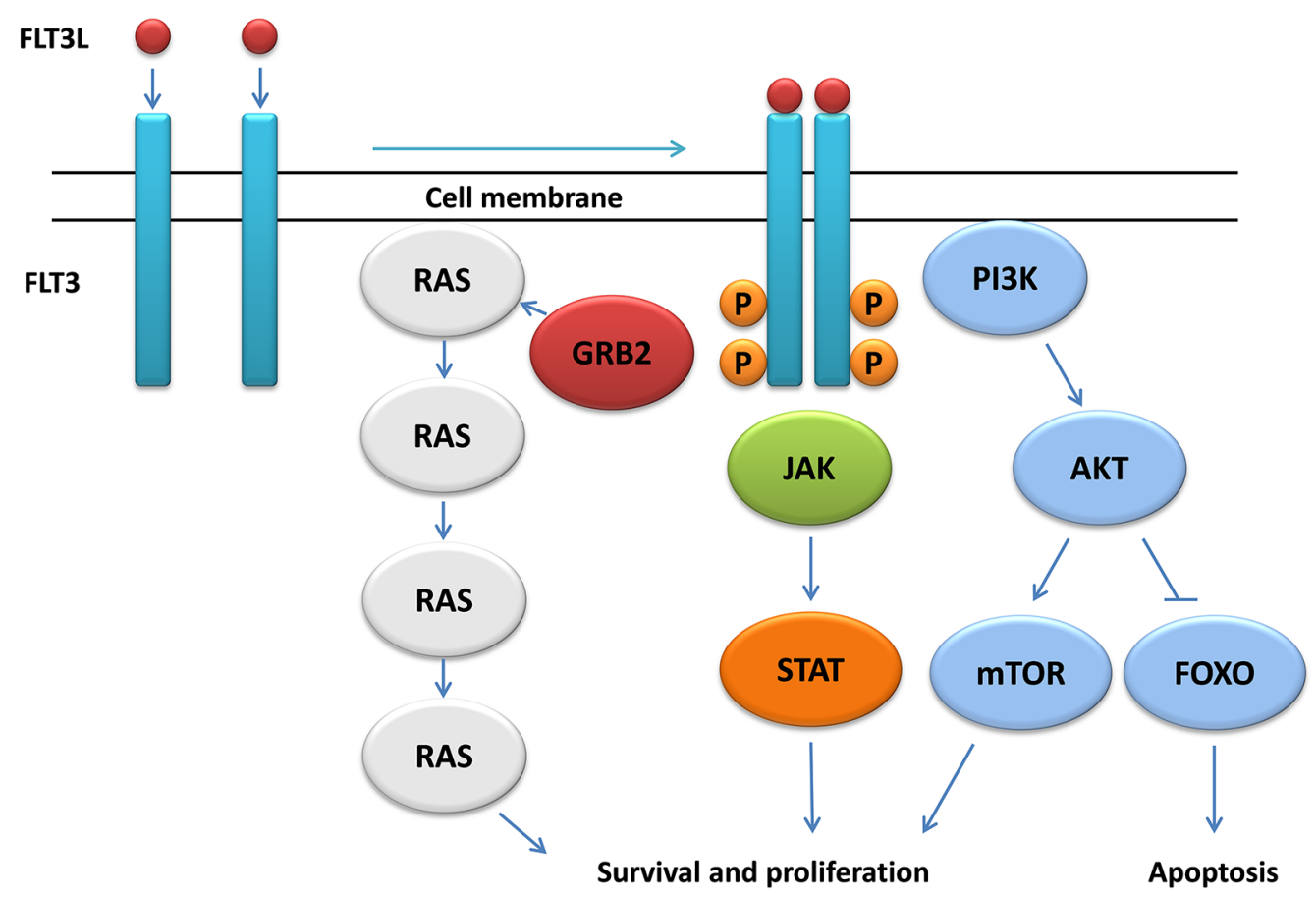

Figure 2 FLT3 signaling pathway. The figure shows the downstream signaling occurs after activation of FLT3. Binding of FLT3 ligand (FLT3L) to FLT3 triggers the dimerization, followed by the activation of the JAK-STAT pathway, the PI3K pathway and the MAPK pathway. These pathways lead to increased cell proliferation/survival and the inhibition of apoptosis.

these cells (24). Over-expression of FLT3 failed to make Ba/ F3 cells cytokine-independent, but the cells over-expressing FLT3 caused leukemia in a mouse engrafted model, suggesting that the leukemogenic role of FLT3 overexpression may need the signaling through FLT3L (23). However, gain-of-function mutant forms of FLT3 are capable of making $\mathrm{Ba} / \mathrm{F} 3$ cells cytokine-independent, indicating their oncogenic potential without ligand engagement (25).

The activating mutations of FLT3 were firstly identified in AML in the year of 1996 (26). They now represent the most frequent molecular abnormalities in AML, making FLT3 an excellent therapeutic target. There are two different types of FLT3 mutations: internal tandem duplications (ITD) and point mutations in the tyrosine kinase domain (TKD) (Figure 3). In newly diagnosed AML patients, the prevalence is $20 \%$ to $30 \%$ for FLT3-ITD and $7 \%$ for FLT3-TKD, both of which can lead to the constitutively activated FLT3 signaling pathway.

FLT3-ITD happens in the form of replicated sequence in the JM domain of FLT3. The duplication varies in their location and length, but always occurs in replicates of three nucleotides and therefore do not affect other regions of FLT3 due to the intact reading frame (26). The in vitro transduction studies indicated that ITD mutations in JM domain promote FLT3 ligand-independent dimerization, autophosphorylation and activation of the receptor (27). In a normal situation, JM domain represses the activation of FLT3 by preventing its dimerization. The presence of ITD disrupts the structure and normal function of JM domain, which leads to the constitutive activation of FLT3. There is evidence showing that FLT3-ITD plays a role in leukemic stem cells (LSCs) since the presence of ITD is found in $\mathrm{CD}_{3} 4^{+} \mathrm{CD} 38^{-}$LSCs (28). Knock-in of FLT3-ITD in murine models results in the enhanced proliferation and survival of multipotent progenitors cells and depletion of long-term hematopoietic stem cells (LT-HSCs) (29). When treated with small molecule FLT3 inhibitor sorafenib, these effects can be reversed, suggesting that the aberrant FLT3-ITD signaling is related to the dysregulation of LSCs in leukemogenesis. FLT3-ITD has significant clinical consequence in AML. The results from a number of studies have revealed that this mutation is associated with disease progression, increased risk of relapse and shorter overall 


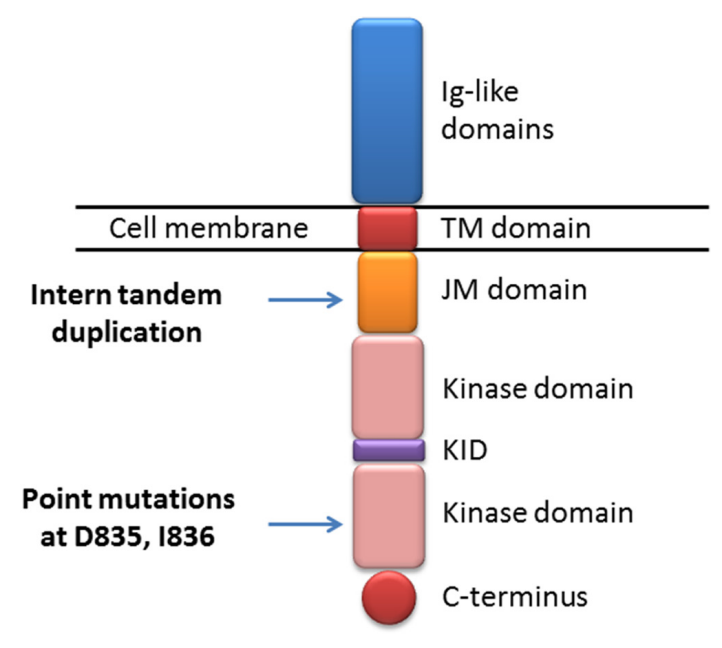

Figure 3 Locations of activating mutations of FLT3. Internal tandem duplication (ITD) occurs in exons 14 or 15 of the JM domain. Point mutations are found in exon 20 of the kinase domain.

survival (30). In addition, the homozygous expression of FLT3-ITD due to loss of heterozygosity (LOH) in the patients leads to a particularly poor outcome, indicating the prognostic significance of FLT3-ITD gene dosage. Although the ITD mutation was mainly found in the JM domain of FLT3, it occurs in another part of the kinase (31). In a study involving $241 \mathrm{AML}$ patients with FLT3-ITD, the ones with ITD in the FLT3 TKD have a significantly worse prognosis, including disease free survival, overall survival and remission rate, compared with the ones with ITD in the JM domain. This indicates ITD location is another important factor related to clinical outcomes (32). In addition, the ITD mutations vary not only in their location but also in their length, and whether their length has clinical significance is still under debate. One study showed that patients with an ITD more than 40 base pairs had a 5 -year OS of $13 \%$, compared to $26 \%$ in those with an ITD shorter than 40 base pairs (33). It was hypothesized that longer ITDs might had enhanced the ability to interrupt the negative regulatory function of the JM domain. But another study drew an opposite conclusion that shorter ITDs have worse prognosis (34). Therefore, further studies are needed to verify the clinical significance of the length of the ITD mutation.

The aberrant activation of signaling pathways led by FLT3-ITD has been reported extensively $(35,36)$. The presence of the ITD in the JM domain leads to constitutive activation of the FLT3, resulting from disruptions of the JM domain structure that normally has a negative regulatory role by keeping FLT3 in the inactive conformation (35). The activation of FLT3 can lead to the activation of the anti-apoptotic PI3K/AKT pathway by phosphorylation of SHP-2, CBL and Gab2. This pathway has been shown constitutively activated in 32D cells transfected by FLT3ITD (37). The MAPK pathway is also constitutively activated by aberrant FLT3-ITD signaling. Both 32D and $\mathrm{Ba} / \mathrm{F} 3$ cells stably expressing FLT3-ITD show activation of MAPK, which is demonstrated by the phosphorylation of ERK1/2 (37). As another important signaling protein, STAT5 is also constitutively phosphorylated and activated in cells expressing FLT3-ITD, including leukemic cell lines and blast cells from AML patients. This transcription factor is actively involved in the regulation of self-renewal and differentiation of hematopoietic progenitor cells and it is only partially activated in FLT3 wild type cells, indicating the alternative signaling pathway induced by FLT3-ITD (38). Besides the over-activation of the above signaling components, suppression of SHP-1 is another potential mechanism underlying ITD-induced leukemogenesis. The SHP-1 phosphatase has a negative regulatory function in multiple growth factors and cytokine signaling pathways. The FLT3-ITD-transfected cells have a threefold decrease in SHP-1 expression, while inhibition of FLT3 by small molecule inhibitor results in the restoration of SHP-1. These data suggest SHP-1 as a tumor suppressor gene in AML containing FLT3-ITD (39). In addition to over-activation of proliferative signaling pathways, FLT3-ITD is also involved in blockage of cell differentiation. Expression of FLT3-ITD prevents the maturing differentiation of $32 \mathrm{D}$ cells into granulocytes when they are stimulated with G-CSF. In these cells, genes facilitating myeloid differentiation are shown to be down-regulated in the presence of FLT3-ITD. As expected, treatment of the cells with a small molecule FLT3 inhibitor restores expression of these genes and leads to the maturing differentiation (40). Even though a great number of evidence supporting FLT3-ITD as a transforming factor in several cytokine-dependent cell lines, it is not sufficient to cause AML in mouse models. Transfection of FLT3-ITD results in cytokine-independent transformation with $\mathrm{Ba} /$ $\mathrm{F} 3$ and 32D cells, and immunodeficient mice injected with these transformed cells develop the phenotypes resembling human acute leukemia. However, in FLT3-ITD-expressing retrovirus-infected bone marrow transplantation model, only a myeloproliferative neoplasm phenotype is observed 
instead of acute leukemia (41). Similarly, the FLT3-ITD knock-in mouse model develops symptoms resembling human CMML, featured by leukocytosis, splenomegaly, and myeloid expansion (42). Interestingly, FLT3-ITD knock-in mice develop acute leukemia when combined with other molecular lesions, including mutant NPM1, NUP98HOXD13D, MLL-AF9 and mutant CEBP $\alpha$ (43-45). These molecular lesions are found in AML patients and coexist with FLT3-ITD sometimes, supporting the model of "multiple hitting" in leukemogenesis. As described above, the gene dose of FLT3-ITD has significant clinical relevance in human AML. Accordingly, homozygous FLT3-ITD knockin mice display a more severe phenotype than heterozygous littermates. In addition, the phenotypes of homozygous mice are irrespective of the presence of FLT3L, supported by crossing FLT3-ITD knock-in mice with FLT3L knockout mice. In contrast, heterozygous mice have decreased severity in their symptoms without FLT3L (46).

FLT3-TKD mutations were firstly reported in the year of 2001, which included mainly missense point mutations on aspartic acid residue D835 and less commonly, isoleucine I836 (47). These TKD mutations can be found in $~ 7 \%$ of de novo AML patients independently of FLT3ITD mutations, and these mutations do not have the same prognostic significance of FLT3-ITD in AML. FLT3TKDs are believed to change the conformation of the TKD of the "inactive state" to the "active state" (48). In wild-type FLT3, the catalytic loop within kinase domain is usually under the "closed" conformation, which prevents binding of ATP. The presence of TKD mutations change the structure into the open conformation and result in the constitutive activation of FLT3. Studies show that the stable expression of FLT3-D835 mutations in 32D and Ba/F3 cells confers these cells cytokine independency (49). The presence of FLT3-D835 mutations leads to constitutive activation of FLT3 and downstream signaling proteins AKT and ERK1/2 in the absence of FLT3L, which is similar to what occurs with FLT-ITD mutants. However, phosphorylation of STAT5 is not as significant as found with FLT3-ITD-transfected cells, indicating the different signaling components are affected by the ITD and TKD mutations (50). Interestingly, in the retrovirus transfected bone marrow transplant model, the TKD mutation leads to lymphoid disorder instead of MPN observed with ITD (50). The leukocytosis and splenomegaly are not present in these mice, but the myeloid expansion was observed. Recently, a knock-in mouse model for the D835Y mutation has been generated (51). Instead of lymphoid disorder, these mice developed MPN phenotypes milder than those found in FLT3-ITD knock-in mice. The survival time is also longer for D835Y knock-in mice compared with FLT3ITD mice. The STAT5 is less phosphorylated in D835Y knock-in mice, which is consistent with the results from cell-based assays. These studies indicate that differential STAT5 signaling may explain the different phenotypes seen between FLT3-TKD and FLT3-ITD leukemia.

\section{FLT3 inhibitors in targeted anti-AML therapies}

FLT3 is an obvious therapeutic target for the development of anti-AML drugs. On the one hand, its gain-of-function mutations frequently occur in AML. On the other hand, the presence of FLT3 mutations confers resistance to conventional chemotherapy and poor prognosis. Therefore, novel therapeutic methods targeting FLT3 are hopeful in improving survival and life quality of AML patients. Small molecule TKIs are the most sought-after targeted therapeutic drugs (52). They are highly effective in treating many types of cancers including chronic myeloid leukemia (CML), non-small cell lung cancer, myelofibrosis, gastrointestinal stromal tumor, renal cell carcinoma, melanoma, hepatocellular carcinoma, and sarcoma (52). Many TKIs for FLT3 have been developed or are under development. It is conceivable that effective low-molecularweight anti-AML drugs will emerge in the near future.

\section{Sorafenib}

Sorafenib is a multi-target TKI that is active against different kinases, including RAF kinase, VEGFR, PDGFR and c-KIT (53). It has been approved for hepatocellular carcinoma, renal cell carcinoma and differentiated thyroid carcinoma. Several studies show that sorafenib is also a potent FLT3 inhibitor with a greater activity against FLT3ITD than the wild type enzyme (54). Phase I clinical trials showed a clinical response in some patients with FLT3ITD when treated with sorafenib as the monotherapy $(55,56)$. The combination of sorafenib with chemotherapy has also been studied in several clinical trials. Although one study reported 93\% FLT3-ITD-positive patients achieved complete remission (57), another phase II trial showed that sorafenib did not benefit AML patients with the overall survival or the event-free survival in combination with " $7+3$ " induction chemotherapy (58). The combination of sorafenib and azacitidine also showed beneficial effects on FLT3-ITD patients in a phase II study (59). Despite the 
mixed results from clinical trials, off-label use of sorafenib for the treatment of FLT3-ITD-positive AML is a common practice in clinics.

\section{Midostaurin}

Midostaurin is another multi-target kinase inhibitor (60). It is a semi-synthetic derivative of staurosporine that was developed originally as a protein kinase $\mathrm{C}$ (PKC) inhibitor but later demonstrated with activity against a member of class III receptor tyrosine kinase including VEGFR, PDGFR, c-KIT and FLT3. In a phase II clinical trial, 20 FLT3-mutation-positive patients with relapsed/ refractory AML or high-grade myelodysplastic syndrome were evaluated for midostaurin monotherapy (61), the clinical response was seen in $70 \%$ patients. In another phase II midostaurin monotherapy study (62), $42 \%$ patients with wild-type FLT3 and 71\% patients with mutant FLT3 showed a clinical response, suggesting the antileukemic activity of midostaurin in AML patients, especially ones with mutant FLT3. The same group evaluated midostaurin combined with chemotherapy in a phase $1 \mathrm{~b}$ clinical trial(63). The complete remission rate was $74 \%$ for patients with wild-type of FLT3 and 92\% for patients with mutant FLT3. In a recently reported phase III trial, midostaurin combined with standard chemotherapy improved overall survival (OS) and event-free survival (EFS) of patients significantly, and the study suggests the use of midostaurin as a component of therapy in patients at a younger age (64).

\section{Lestaurtinib}

Lestaurtinib is also a kinase inhibitor with a broad spectrum of inhibitory activity. Like midostaurin, lestaurtinib is structurally related to staurosporine. It has potent activity against FLT3, JAK2, TrkA, TrkB and TrkC. Lestaurtinib has been shown to inhibit wild-type and mutant FLT3 in vitro and prolongs survival in the mouse xenograft model (65). In a phase I/II clinical trial, lestaurtinib as a single-agent led to a reduction of blasts in both peripheral blood and bone marrow in 36\% AML patients with mutant FLT3 (66). This study was followed by a phase 2 trial by the same group, in which the clinical response was observed in AML patients irrespective of FLT3 mutation status (67). The clinical effects of lestaurtinib plus chemotherapy were assessed in a randomized phase III clinical trial (68). Unfortunately, no difference was detected between patients treated with chemotherapy alone or followed by lestaurtinib in term of complete remission or overall survival. This indicates that lestaurtinib treatment after chemotherapy does not benefit AML patients with mutant FLT3, which may be due to pharmacokinetic properties of the compound. In another clinical study of lestaurtinib combined with chemotherapy (69), no significant differences were seen in either 5 -year overall survival or 5-year relapse-free survival, but the subgroup of patients showing $>85 \%$ FLT3 inhibition had improved survival, suggesting sustained FLT3 inhibition is important.

\section{Sunitinib}

Sunitinib is an oral-available small molecule TKI that is approved for the treatment of renal cell carcinoma, gastrointestinal stromal tumor and pancreatic neuroendocrine tumor (70). Its targets include FLT3, PDGFR, VEGFR and c-KIT. In preclinical studies, sunitinib was shown to have potent antileukemic activity in multiple xenograft mouse models and the bone marrow transplant model (71). Two phase I clinical trials assessing sunitinib as monotherapy in AML patients were performed $(72,73)$. In both studies, clinical responses including short duration of partial remissions were observed. Following these phase I studies, one group undertook a phase II study to evaluate the combination of sunitinib with induction chemotherapy in FLT3-mutant AML patients older than 60 years. The rate of complete remission was $59 \%$ in all patients enrolled, 53\% in FLT3-ITD positive patients and $71 \%$ in patients with FLT3-TKD mutations (74). This suggests the treatment combination benefits AML patients and further studies involving more patients are warranted.

\section{Quizartinib}

Quizartinib was specifically designed as an FLT3 inhibitor with high potency and selectivity. In mouse xenograft model, quizartinib significantly extends the life span at a dose as low as $1 \mathrm{mg} / \mathrm{kg}$ daily and eradicates tumors at $10 \mathrm{mg} / \mathrm{kg}$ (75). In a phase I clinical trial, $30 \%$ patients irrespective of FLT3 mutant status showed clinical response (76). In patients with FLT3-ITD, 53\% showed a clinical response compared with $14 \%$ in patients with wildtype FLT3. Furthermore, the complete inhibition of FLT3ITD was observed based on in vitro plasma inhibitory assays. A subsequent phase II study was performed. A total of 333 patients were included in this study. Promisingly, 44\% patients with FLT-ITD and $34 \%$ patients with wild-type 
of FLT3 achieved the composite complete remission (77). Phase III trial designated QuANTUM is ongoing to test quizartinib monotherapy and in combination with the standard induction chemotherapy (78).

\section{Crenolanib}

Crenolanib is a small molecule TKI originally developed as PDGFR inhibitor. In the preclinical studies including in vitro binding assays, cell lines and mouse xenograft models, crenolanib showed potent activity against both FLT3-ITD and FLT3-TKD. This property is promising considering some FLT3-TKD mutants such as D835 resist the inhibition of most currently available FLT3 inhibitors (79). In the previous clinical trials evaluating crenolanib as PDGFR inhibitor, its safety, tolerability and pharmacokinetics have been well described, which facilitates clinical trials of crenolanib as an FLT3 inhibitor in AML patients (80). In one study, Mark Levis and colleagues have demonstrated the concentration of crenolanib in the plasma of patients collected from clinical trials is sufficient to inhibit both FLT3-ITD and resistance-conferring FLT3-D835 mutants (81). In a recent phase II clinical trial, $41 \%$ patients enrolled achieved response (82). Other phase I/II studies assessing the combination of crenolanib with induction chemotherapy are undergoing.

\section{Gilteritinib}

Gilteritinib is a pyrazinecarboxamide derivative with the properties of selectivity, potency, and activity against various FLT3-activating mutations (83). In a phase I/II study, $46 \%$ patients achieved complete remission irrespective of FLT3 mutation status. Importantly, 29\% of patients with FLT3/ TKD mutations also achieved complete remission $(84,85)$. Other clinical trials assessing the combination of gilteritinib and chemotherapy are ongoing (86).

\section{Novel FLT3 inbibitors in preclinical stages}

Studies from clinical trials above suggest that newer generation of FLT3 inhibitors produced more promising results compared with the first generation FLT3 inhibitors. This is due to their improved properties including potency, selectivity and extended activity against FLT3-TKD. Adding excitement to the development, several novel inhibitors against FLT3 have been developed and investigated in preclinical stages. These small molecules are more specific and potent, and some of them effectively target additional FLT3 mutations, especially FLT3 D835 in vitro and in vivo (87-95). JI6 represents such an example. It inhibits both FLT3-ITD and FLT3-D835 mutants with equal high potency, and oral administration of the compound into xenograft mouse models significantly inhibited tumor cell growth and expanded the lifespan of the mice (93). Therefore, clinical trials of these novel inhibitors may be warranted. One should expect better clinical efficacy and less toxicity from newer FLT3 inhibitors.

\section{Mechanisms of drug resistance}

During clinical studies, resistance to the treatment with FLT3 inhibitors has been observed in a great number of patients. There are two potential mechanisms underlying the drug resistance. One comes from the inherent nature of leukemic blasts and the drugs used, and the other is caused by the acquired feature of the cancer cells during the drug treatment. Both inherent and acquired resistance mechanisms will be discussed below.

\section{Inberent mechanisms}

Existing FLT3 inhibitors exhibit a different spectrum of activities against FLT3 mutants. Preclinical studies have demonstrated that certain FLT3-TKD mutants are inherently resistant to FLT3 inhibitors (96). For instance, $\mathrm{Ba} / \mathrm{F} 3$ cells transformed by the FLT3-D835Y mutant are resistant to the treatment with sorafenib and quizartinib. This phenomenon was also observed in clinical trials. Patients with FLT3-TKD mutations on D835 indeed had a reduced response to these two drugs $(48,97)$. Furthermore, within patients containing FLT3-ITD mutations, the location and length of ITD also affect the sensitivity to FLT3 inhibitor treatment of FLT3 inhibitors $(32,33,98,99)$. Patients with ITD reaching to the TKD away from the JM domain are known to have a poor prognosis for the induction chemotherapy $(32,33)$. Further studies demonstrated that this type of ITD mutations also conferred resistance to FLT3 inhibitor therapies, at least due to up-regulation of MCL-1 $(98,99)$. The length of ITD correlates with resistance to FLT3 inhibitors as well, since longer ITDs are more likely to invade the TKD. This upfront type of drug resistance due to the narrow activity of inhibitors can presumably be overcome by the generation of FLT3 inhibitors with broader inhibitory activities toward various types of FLT3 mutants. However, persistent 
activation of FLT3 downstream signaling pathways also contributes to inherent resistance to FLT3 inhibitors since constitutive activation of MAPK and STAT5 pathways was observed even when FLT3 was completely inhibited in some cases (100). Whether this is caused by other molecular lesions in addition to FLT3 mutations awaits further investigation.

Insufficient drug concentrations in the plasma may represent another reason conferring the drug resistance (68). Some FLT3 inhibitors can be rapidly metabolized in the liver by specific enzymes. Although there are drugs that can slow down the metabolism of FLT3 inhibitors, the subsequent side effects are hard to predict. In addition, some FLT3 inhibitors are sequestered serum proteins such as an alpha-1 acid glycoprotein, and their free concentrations are reduced in plasma. Finally, the microenvironment or niche where leukemia cancer stem cells reside also contributes to the development of drug resistance (101). While blasts in the circulation are readily susceptible to FLT3 inhibitors, those in the bone marrow may be protected by stromal cells. This is supported by the fact that quizartinib has cytotoxic effects on peripheral blasts but only induces differentiation of bone marrow blasts (102).

\section{Acquired resistance}

Some FLT3-TKD mutations arise in response to the treatment of FLT3 inhibitor (48). These mutations mainly involve D835 and F691L, which may be pre-existing as a small subclone and selected upon the treatment with FLT3 inhibitors targeting FLT3-ITD mutations. This type of emerging inhibitor-resistant mutations is also seen in many other types of cancers and represents a common mechanism underlying resistance to targeted therapies. For instance, T315I mutation of BCR-ABL confers resistance to imatinib treatment in CML patients. But the next generation of BCR-ABL inhibitor ponatinib can overcome this resistance since it effectively inhibits BCR-ABLT315I. Therefore, identifying novel inhibitors that can effectively target known resistance-conferring mutations in FLT3 is important in the development of targeted drug therapies to treat AML.

Autocrine FLT3L stimulation is another resistance mechanism (103). In cell-based assays, the increased expression of FLT3L was revealed in FLT3 mutant MV4-11 cells treated with FLT3 inhibitors. In clinical trials, it was observed that chemotherapy could also result in increased concentrations of FLT3L, which potentially decrease the effectiveness of subsequent treatment FLT3 inhibition therapies. This has brought a major challenge to the combination therapy involving FLT3 inhibitors and cytotoxic chemotherapy.

Acquisition of other gene mutations and activation of alternative signaling pathways are also seen during treatment with FLT3 inhibitors $(104,105)$. When treated with increasing concentrations of lestaurtinib, FLT3-ITDpositive Molm-14 cells gained an NRAS mutation that constitutively activates AKT and MAPK pathways. RAS mutations have been found in relapsed patients with FLT3mutant-positive AML. In addition, mutations or aberrant expressions of genes including BCL2, NF-kB and MCL-1 have also been reported and are believed to be responsible for the FLT3 inhibitor resistance. Therefore, combinations of FLT3 inhibitors with other targeted therapies are interesting topics to be explored in the future $(106,107)$.

\section{Conclusions}

Mutated in a significant portion of AML cases and representing a primary cause of leukemogenesis, FLT3 is an obvious target for therapeutic drug development. However, after many years of intensive studies, challenges remain in the development of FLT3 inhibitors as therapeutic antiAML drugs. More potent FLT3 inhibitors with broader inhibitory effects on multiple FLT3 mutations are highly desirable. Combination drug therapies targeting multiple pathways especially those related to cancer stem cells are required to eradicate AML. In this regards, further studies on the involvement of various gene mutations in the pathogenesis of AML are warranted. Above all, for the development of effective anti-AML therapies, FLT3 can be targeted and has to be targeted. It will be a matter of time before a highly effective anti-AML drug targeting FLT3 emerges.

\section{Acknowledgements}

None.

\section{Footnote}

Conflicts of Interest: The authors have no conflicts of interest to declare.

\section{References}

1. Estey E, Dohner H. Acute myeloid leukaemia. Lancet 
2006;368:1894-907.

2. Howlader N NA, Krapcho M, Miller D, Bishop K, Altekruse SF, Kosary CL, Yu M, Ruhl J, Tatalovich Z, Mariotto A, Lewis DR, Chen HS, Feuer EJ, Cronin KA SEER Cancer Statistics Review, 1975-2013. National Cancer Institute 2016;Bethesda, MD, http://seer.cancer. gov/csr/1975_2013/, based on November 2015 SEER data submission, posted to the SEER web site, April 2016.

3. Glick AD, Paniker K, Flexner JM, et al. Acute leukemia of adults. Ultrastructural, cytochemical and histologic observations in 100 cases. Am J Clin Pathol 1980;73:459-70.

4. Kaushansky K, Lichtman MA, Prchal J, et al. Levi, Oliver Press, Linda Burns, Michael Caligiuri. Williams hematology. McGraw-Hill Med, 2015.

5. Hofmann WK, Stauch M, Hoffken K. Impaired granulocytic function in patients with acute leukaemia: only partial normalisation after successful remissioninducing treatment. J Cancer Res Clin Oncol 1998;124:113-6.

6. Burns CP, Armitage JO, Frey AL, et al. Analysis of the presenting features of adult acute leukemia: the FrenchAmerican-British classification. Cancer 1981;47:2460-9.

7. Kaplan SS, Penchansky L, Krause JR, et al. Simultaneous evaluation of terminal deoxynucleotidyl transferase and myeloperoxidase in acute leukemias using an immunocytochemical method. Am J Clin Pathol 1987;87:732-8.

8. Bene MC, Castoldi G, Knapp W, et al. Proposals for the immunological classification of acute leukemias. European Group for the Immunological Characterization of Leukemias (EGIL). Leukemia 1995;9:1783-6.

9. Dombret H, Gardin C. An update of current treatments for adult acute myeloid leukemia. Blood 2016;127:53-61.

10. Estey EH. Acute myeloid leukemia: 2014 update on risk-stratification and management. Am J Hematol 2014;89:1063-81.

11. Rosnet O, Stephenson D, Mattei MG, et al. Close physical linkage of the FLT1 and FLT3 genes on chromosome 13 in man and chromosome 5 in mouse. Oncogene 1993;8:173-9.

12. Agnes F, Shamoon B, Dina C, et al. Genomic structure of the downstream part of the human FLT3 gene: exon/ intron structure conservation among genes encoding receptor tyrosine kinases (RTK) of subclass III. Gene 1994;145:283-8.

13. Lyman SD, James L, Zappone J, et al. Characterization of the protein encoded by the flt 3 (flk2) receptor-like tyrosine kinase gene. Oncogene 1993;8:815-22.
14. Carow CE, Levenstein M, Kaufmann SH, et al. Expression of the hematopoietic growth factor receptor FLT3 (STK-1/ Flk2) in human leukemias. Blood 1996;87:1089-96.

15. Rosnet O, Schiff C, Pebusque MJ, et al. Human FLT3/FLK2 gene: cDNA cloning and expression in hematopoietic cells. Blood 1993;82:1110-9.

16. Mackarehtschian K, Hardin JD, Moore KA, et al. Targeted disruption of the $\mathrm{flk} 2 / \mathrm{flt} 3$ gene leads to deficiencies in primitive hematopoietic progenitors. Immunity 1995;3:147-61.

17. Gotze KS, Ramirez M, Tabor K, et al. Flt3high and Flt3low CD34+ progenitor cells isolated from human bone marrow are functionally distinct. Blood 1998;91:1947-58.

18. Lyman SD. Biology of flt 3 ligand and receptor. Int J Hematol 1995;62:63-73.

19. Rosnet O, Buhring HJ, deLapeyriere O, et al. Expression and signal transduction of the FLT3 tyrosine kinase receptor. Acta Haematol 1996;95:218-23.

20. Lavagna-Sevenier C, Marchetto S, Birnbaum D, et al. FLT3 signaling in hematopoietic cells involves CBL, SHC and an unknown P115 as prominent tyrosinephosphorylated substrates. Leukemia 1998;12:301-10.

21. Cook AM, Li L, Ho Y, et al. Role of altered growth factor receptor-mediated JAK2 signaling in growth and maintenance of human acute myeloid leukemia stem cells. Blood 2014;123:2826-37.

22. Zhang S, Broxmeyer HE. p85 subunit of PI3 kinase does not bind to human Flt3 receptor, but associates with SHP2, SHIP, and a tyrosine-phosphorylated 100-kDa protein in Flt3 ligand-stimulated hematopoietic cells. Biochem Biophys Res Commun 1999;254:440-5.

23. Zhang S, Mantel C, Broxmeyer HE. Flt3 signaling involves tyrosyl-phosphorylation of SHP-2 and SHIP and their association with Grb2 and Shc in Baf3/Flt3 cells. J Leukoc Biol 1999;65:372-80.

24. Meierhoff G, Dehmel U, Gruss HJ, et al. Expression of FLT3 receptor and FLT3-ligand in human leukemialymphoma cell lines. Leukemia 1995;9:1368-72.

25. Hayakawa F, Towatari M, Kiyoi H, et al. Tandemduplicated Flt3 constitutively activates STAT5 and MAP kinase and introduces autonomous cell growth in IL-3dependent cell lines. Oncogene 2000;19:624-31.

26. Nakao M, Yokota S, Iwai T, et al. Internal tandem duplication of the flt 3 gene found in acute myeloid leukemia. Leukemia 1996;10:1911-8.

27. Kiyoi H, Ohno R, Ueda R, et al. Mechanism of constitutive activation of FLT3 with internal tandem duplication in the juxtamembrane domain. Oncogene 
2002;21:2555-63.

28. Levis M, Murphy KM, Pham R, et al. Internal tandem duplications of the FLT3 gene are present in leukemia stem cells. Blood 2005;106:673-80.

29. Chu SH, Heiser D, Li L, et al. FLT3-ITD knockin impairs hematopoietic stem cell quiescence/homeostasis, leading to myeloproliferative neoplasm. Cell Stem Cell 2012;11:346-58.

30. Levis M, Small D. FLT3: ITDoes matter in leukemia. Leukemia 2003;17:1738-52.

31. Breitenbuecher F, Schnittger S, Grundler R, et al. Identification of a novel type of ITD mutations located in nonjuxtamembrane domains of the FLT3 tyrosine kinase receptor. Blood 2009;113:4074-7.

32. Kayser S, Schlenk RF, Londono MC, et al. Insertion of FLT3 internal tandem duplication in the tyrosine kinase domain-1 is associated with resistance to chemotherapy and inferior outcome. Blood 2009;114:2386-92.

33. Stirewalt DL, Kopecky KJ, Meshinchi S, et al. Size of FLT3 internal tandem duplication has prognostic significance in patients with acute myeloid leukemia. Blood 2006;107:3724-6.

34. Gale RE, Green C, Allen C, et al. The impact of FLT3 internal tandem duplication mutant level, number, size, and interaction with NPM1 mutations in a large cohort of young adult patients with acute myeloid leukemia. Blood 2008;111:2776-84.

35. Hubbard SR. Juxtamembrane autoinhibition in receptor tyrosine kinases. Nat Rev Mol Cell Biol 2004;5:464-71.

36. Mol CD, Dougan DR, Schneider TR, et al. Structural basis for the autoinhibition and STI-571 inhibition of c-Kit tyrosine kinase. J Biol Chem 2004;279:31655-63.

37. Mizuki M, Fenski R, Halfter H, et al. Flt3 mutations from patients with acute myeloid leukemia induce transformation of 32D cells mediated by the Ras and STAT5 pathways. Blood 2000;96:3907-14.

38. Zhang S, Fukuda S, Lee Y, et al. Essential role of signal transducer and activator of transcription (Stat)5a but not Stat5b for Flt3-dependent signaling. J Exp Med 2000;192:719-28.

39. Chen P, Levis M, Brown P, et al. FLT3/ITD mutation signaling includes suppression of SHP-1. J Biol Chem 2005;280:5361-9.

40. Zheng R, Friedman AD, Levis M, et al. Internal tandem duplication mutation of FLT3 blocks myeloid differentiation through suppression of C/EBPalpha expression. Blood 2004;103:1883-90.

41. Kelly LM, Liu Q, Kutok JL, et al. FLT3 internal tandem duplication mutations associated with human acute myeloid leukemias induce myeloproliferative disease in a murine bone marrow transplant model. Blood 2002;99:310-8.

42. Lee BH, Tothova Z, Levine RL, et al. FLT3 mutations confer enhanced proliferation and survival properties to multipotent progenitors in a murine model of chronic myelomonocytic leukemia. Cancer Cell 2007;12:367-80.

43. Stubbs MC, Kim YM, Krivtsov AV, et al. MLL-AF9 and FLT3 cooperation in acute myelogenous leukemia: development of a model for rapid therapeutic assessment. Leukemia 2008;22:66-77.

44. Reckzeh K, Bereshchenko O, Mead A, et al. Molecular and cellular effects of oncogene cooperation in a genetically accurate AML mouse model. Leukemia 2012;26:1527-36.

45. Rau R, Magoon D, Greenblatt S, et al. NPMc+ cooperates with Flt3/ITD mutations to cause acute leukemia recapitulating human disease. Exp Hematol 2014;42:101-13 e5.

46. Kharazi S, Mead AJ, Mansour A, et al. Impact of gene dosage, loss of wild-type allele, and FLT3 ligand on Flt3ITD-induced myeloproliferation. Blood 2011;118:3613-21.

47. Abu-Duhier FM, Goodeve AC, Wilson GA, et al. Identification of novel FLT-3 Asp835 mutations in adult acute myeloid leukaemia. Br J Haematol 2001;113:983-8.

48. Smith CC, Wang Q, Chin CS, et al. Validation of ITD mutations in FLT3 as a therapeutic target in human acute myeloid leukaemia. Nature 2012;485:260-3.

49. Yamaguchi H, Hanawa H, Uchida N, et al. Multistep pathogenesis of leukemia via the MLL-AF4 chimeric gene/Flt3 gene tyrosine kinase domain (TKD) mutationrelated enhancement of S100A6 expression. Exp Hematol 2009;37:701-14.

50. Grundler R, Miething C, Thiede C, et al. FLT3-ITD and tyrosine kinase domain mutants induce 2 distinct phenotypes in a murine bone marrow transplantation model. Blood 2005;105:4792-9.

51. Bailey E, Li L, Duffield AS, et al. FLT3/D835Y mutation knock-in mice display less aggressive disease compared with FLT3/internal tandem duplication (ITD) mice. Proc Natl Acad Sci U S A 2013;110:21113-8.

52. Zhang J, Yang PL, Gray NS. Targeting cancer with small molecule kinase inhibitors. Nat Rev Cancer 2009;9:28-39.

53. Hasskarl J. Sorafenib. Recent Results Cancer Res 2010;184:61-70.

54. O'Farrell AM, Abrams TJ, Yuen HA, et al. SU11248 is a novel FLT3 tyrosine kinase inhibitor with potent activity in vitro and in vivo. Blood 2003;101:3597-605. 
55. Zhang W, Konopleva M, Shi YX, et al. Mutant FLT3: a direct target of sorafenib in acute myelogenous leukemia. J Natl Cancer Inst 2008;100:184-98.

56. Crump M, Hedley D, Kamel-Reid S, et al. A randomized phase I clinical and biologic study of two schedules of sorafenib in patients with myelodysplastic syndrome or acute myeloid leukemia: a NCIC (National Cancer Institute of Canada) Clinical Trials Group Study. Leuk Lymphoma 2010;51:252-60.

57. Ravandi F, Cortes JE, Jones D, et al. Phase I/II study of combination therapy with sorafenib, idarubicin, and cytarabine in younger patients with acute myeloid leukemia. J Clin Oncol 2010;28:1856-62.

58. Serve H, Krug U, Wagner R, et al. Sorafenib in combination with intensive chemotherapy in elderly patients with acute myeloid leukemia: results from a randomized, placebo-controlled trial. J Clin Oncol 2013;31:3110-8.

59. Ravandi F, Alattar ML, Grunwald MR, et al. Phase 2 study of azacytidine plus sorafenib in patients with acute myeloid leukemia and FLT-3 internal tandem duplication mutation. Blood 2013;121:4655-62.

60. Fabbro D, Ruetz S, Bodis S, et al. PKC412--a protein kinase inhibitor with a broad therapeutic potential. Anticancer Drug Des 2000;15:17-28.

61. Stone RM, DeAngelo DJ, Klimek V, et al. Patients with acute myeloid leukemia and an activating mutation in FLT3 respond to a small-molecule FLT3 tyrosine kinase inhibitor, PKC412. Blood 2005;105:54-60.

62. Fischer T, Stone RM, Deangelo DJ, et al. Phase IIB trial of oral Midostaurin (PKC412), the FMS-like tyrosine kinase 3 receptor (FLT3) and multi-targeted kinase inhibitor, in patients with acute myeloid leukemia and high-risk myelodysplastic syndrome with either wild-type or mutated FLT3. J Clin Oncol 2010;28:4339-45.

63. Stone RM, Fischer T, Paquette R, et al. Phase IB study of the FLT3 kinase inhibitor midostaurin with chemotherapy in younger newly diagnosed adult patients with acute myeloid leukemia. Leukemia 2012;26:2061-8.

64. Richard M, Stone SM, Sanford BL, et al. The MultiKinase Inhibitor Midostaurin (M) Prolongs Survival Compared with Placebo $(\mathrm{P})$ in Combination with Daunorubicin (D)/Cytarabine (C) Induction (ind), HighDose C Consolidation (consol), and As Maintenance (maint) Therapy in Newly Diagnosed Acute Myeloid Leukemia (AML) Patients (pts) Age 18-60 with FLT3 Mutations (muts): An International Prospective Randomized (rand) P-Controlled Double-Blind Trial
(CALGB 10603/RATIFY [Alliance]). Blood 2015;126.

65. Levis M, Allebach J, Tse KF, et al. A FLT3-targeted tyrosine kinase inhibitor is cytotoxic to leukemia cells in vitro and in vivo. Blood 2002;99:3885-91.

66. Smith BD, Levis M, Beran M, et al. Single-agent CEP701, a novel FLT3 inhibitor, shows biologic and clinical activity in patients with relapsed or refractory acute myeloid leukemia. Blood 2004;103:3669-76.

67. Knapper S, Burnett AK, Littlewood T, et al. A phase 2 trial of the FLT3 inhibitor lestaurtinib (CEP701) as first-line treatment for older patients with acute myeloid leukemia not considered fit for intensive chemotherapy. Blood 2006;108:3262-70.

68. Levis M, Ravandi F, Wang ES, et al. Results from a randomized trial of salvage chemotherapy followed by lestaurtinib for patients with FLT3 mutant AML in first relapse. Blood 2011;117:3294-301.

69. Knapper S, Russell N, Gilkes A, et al. A randomized assessment of adding the kinase inhibitor lestaurtinib to first-line chemotherapy for FLT3-mutated AML. Blood 2017;129:1143-54.

70. Chow LQ, Eckhardt SG. Sunitinib: from rational design to clinical efficacy. J Clin Oncol 2007;25:884-96.

71. Deeks ED, Keating GM. Sunitinib. Drugs 2006;66:225566; discussion 2267-8.

72. O'Farrell AM, Foran JM, Fiedler W, et al. An innovative phase I clinical study demonstrates inhibition of FLT3 phosphorylation by SU11248 in acute myeloid leukemia patients. Clin Cancer Res 2003;9:5465-76.

73. Fiedler $W$, Serve H, Dohner H, et al. A phase 1 study of SU11248 in the treatment of patients with refractory or resistant acute myeloid leukemia (AML) or not amenable to conventional therapy for the disease. Blood 2005;105:986-93.

74. Fiedler W, Kayser S, Kebenko M, et al. A phase I/II study of sunitinib and intensive chemotherapy in patients over 60 years of age with acute myeloid leukaemia and activating FLT3 mutations. Br J Haematol 2015;169:694-700.

75. Zarrinkar PP, Gunawardane RN, Cramer MD, et al. AC220 is a uniquely potent and selective inhibitor of FLT3 for the treatment of acute myeloid leukemia (AML). Blood 2009;114:2984-92.

76. Cortes JE, Kantarjian H, Foran JM, et al. Phase I study of quizartinib administered daily to patients with relapsed or refractory acute myeloid leukemia irrespective of FMS-like tyrosine kinase 3-internal tandem duplication status. J Clin Oncol 2013;31:3681-7.

77. Grunwald MR, Levis MJ. FLT3 inhibitors for acute 
myeloid leukemia: a review of their efficacy and mechanisms of resistance. Int J Hematol 2013;97:683-94.

78. Harry Paul Erba MJL, Mikkael A. Sekeres, Herve Dombret, Sergio Amadori, Oleg Zernovak, Derek Edward Mires, NanXiang Ge, James Hanyok. Phase 3 (P3) study of quizartinib (Q) or placebo (P) with induction (IND) and consolidation chemotherapy (CON) and as maintenance $(\mathrm{MN})$ in patients (pts) with newly diagnosed (NDx) FLT3-ITD-positive acute myeloid leukemia (AML): the QuANTUM-First study. J Clin Oncol 2016;34.

79. Zimmerman EI, Turner DC, Buaboonnam J, et al. Crenolanib is active against models of drug-resistant FLT3-ITD-positive acute myeloid leukemia. Blood 2013;122:3607-15.

80. Smith CC, Lasater EA, Lin KC, et al. Crenolanib is a selective type I pan-FLT3 inhibitor. Proc Natl Acad Sci U S A 2014;111:5319-24.

81. Lewis NL, Lewis LD, Eder JP, et al. Phase I study of the safety, tolerability, and pharmacokinetics of oral CP868,596 , a highly specific platelet-derived growth factor receptor tyrosine kinase inhibitor in patients with advanced cancers. J Clin Oncol 2009;27:5262-9.

82. Jorge E. Cortes HMK, Tapan M. Kadia, Gautam Borthakur, Marina Konopleva, Guillermo Garcia-Manero, Naval Guastad Daver. Crenolanib besylate, a type I panFLT3 inhibitor, to demonstrate clinical activity in multiply relapsed FLT3-ITD and D835 AML. J Clin Oncol $2016 ; 34$.

83. Lee LY, Hernandez D, Rajkhowa T, et al. Preclinical studies of gilteritinib, a next-generation FLT3 inhibitor. Blood 2017;129:257-60.

84. Catherine C, Smith MJ, Litzow MR, et al. Pharmacokinetic Profile and Pharmacodynamic Effects of ASP2215, a Selective, Potent Inhibitor of FLT3/AXL, in Patients with Relapsed or Refractory Acute Myeloid Leukemia: Results from a First-in-Human Phase 1/2 Study. Blood 2015;126:4836.

85. Jessica JK, Altman AE, Jorge JE, et al. Antileukemic Activity and Tolerability of ASP2215 80mg and Greater in FLT3 Mutation-Positive Subjects with Relapsed or Refractory Acute Myeloid Leukemia: Results from a Phase 1/2, Open-Label, Dose-Escalation/Dose-Response Study. Blood 2015;126:321.

86. Alexander E. Perl JEC, Stephen Anthony Strickland, Ellen K. Ritchie, Andreas Neubauer, Giovanni Martinelli, Tomoki Naoe, Arnaud Pigneux, Philippe H. Rousselot, Christoph Röllig. A phase 3, open-label, randomized study of the FLT3 inhibitor gilteritinib versus salvage chemotherapy in adults with first relapse or primary refractory FLT3 mutation-positive acute myeloid leukemia. J Clin Oncol 2016;34.

87. Ma F, Liu P, Lei M, et al. Design, synthesis and biological evaluation of indolin-2-one-based derivatives as potent, selective and efficacious inhibitors of FMS-like tyrosine kinase3 (FLT3). Eur J Med Chem 2017;127:72-86.

88. Kowolik CM, Lin M, Xie J, et al. NT1721, a novel epidithiodiketopiperazine, exhibits potent in vitro and in vivo efficacy against acute myeloid leukemia. Oncotarget 2016;7:86186-97.

89. Sun D, Yang Y, Lyu J, et al. Discovery and Rational Design of Pteridin-7(8H)-one-Based Inhibitors Targeting FMSlike Tyrosine Kinase 3 (FLT3) and Its Mutants. J Med Chem 2016;59:6187-200.

90. Zhang W, Borthakur G, Gao C, et al. The Dual MEK/ FLT3 Inhibitor E6201 Exerts Cytotoxic Activity against Acute Myeloid Leukemia Cells Harboring ResistanceConferring FLT3 Mutations. Cancer Res 2016;76:1528-37.

91. Li X, Wang A, Yu K, et al. Discovery of (R)-1-(3(4-Amino-3-(4-phenoxyphenyl)-1H-pyrazolo[3,4-d] pyrimidin-1-yl)piperidin- 1-yl)-2-(dimethylamino) ethanone (CHMFL-FLT3-122) as a Potent and Orally Available FLT3 Kinase Inhibitor for FLT3-ITD Positive Acute Myeloid Leukemia. J Med Chem 2015;58:9625-38.

92. Ma S, Yang LL, Niu T, et al. SKLB-677, an FLT3 and Wnt/beta-catenin signaling inhibitor, displays potent activity in models of FLT3-driven AML. Sci Rep 2015;5:15646.

93. Chen Y, Guo Y, Zhao W, et al. Identification of an orally available compound with potent and broad FLT3 inhibition activity. Oncogene 2016;35:2971-8.

94. Guo Y, Chen Y, Xu X, et al. SU11652 Inhibits tyrosine kinase activity of FLT3 and growth of MV-4-11 cells. J Hematol Oncol 2012;5:72.

95. Pawar R, Bali OP, Malhotra BK, et al. Recent advances and novel agents for FLT3 mutated acute myeloid leukemia. Stem Cell Investig 2014;1:7.

96. Clark JJ, Cools J, Curley DP, et al. Variable sensitivity of FLT3 activation loop mutations to the small molecule tyrosine kinase inhibitor MLN518. Blood 2004;104:2867-72.

97. Baker SD, Zimmerman EI, Wang YD, et al. Emergence of polyclonal FLT3 tyrosine kinase domain mutations during sequential therapy with sorafenib and sunitinib in FLT3ITD-positive acute myeloid leukemia. Clin Cancer Res 2013;19:5758-68.

98. Breitenbuecher F, Markova B, Kasper S, et al. A novel 
molecular mechanism of primary resistance to FLT3kinase inhibitors in AML. Blood 2009;113:4063-73.

99. Schiller J, Praulich I, Krings Rocha C, et al. Patientspecific analysis of FLT3 internal tandem duplications for the prognostication and monitoring of acute myeloid leukemia. Eur J Haematol 2012;89:53-62.

100.Knapper S, Mills KI, Gilkes AF, et al. The effects of lestaurtinib (CEP701) and PKC412 on primary AML blasts: the induction of cytotoxicity varies with dependence on FLT3 signaling in both FLT3-mutated and wild-type cases. Blood 2006;108:3494-503.

101. Kindler T, Lipka DB, Fischer T. FLT3 as a therapeutic target in AML: still challenging after all these years. Blood 2010;116:5089-102.

102. Sexauer A, Perl A, Yang X, et al. Terminal myeloid differentiation in vivo is induced by FLT3 inhibition in FLT3/ITD AML. Blood 2012;120:4205-14.

103.Sato T, Yang X, Knapper S, et al. FLT3 ligand impedes

doi: $10.21037 /$ sci.2017.05.04

Cite this article as: Chen Y, Pan Y, Guo Y, Zhao W, Ho WT, Wang J, Xu M, Yang FC, Zhao ZJ. Tyrosine kinase inhibitors targeting FLT3 in the treatment of acute myeloid leukemia. Stem Cell Investig 2017;4:48. the efficacy of FLT3 inhibitors in vitro and in vivo. Blood 2011;117:3286-93.

104. Nakano Y, Kiyoi H, Miyawaki S, et al. Molecular evolution of acute myeloid leukaemia in relapse: unstable N-ras and FLT3 genes compared with p53 gene. Br J Haematol 1999;104:659-64.

105.Piloto O, Wright M, Brown P, et al. Prolonged exposure to FLT3 inhibitors leads to resistance via activation of parallel signaling pathways. Blood 2007;109:1643-52.

106. Pleyer L, Burgstaller S, Stauder R, et al. Azacitidine frontline in 339 patients with myelodysplastic syndromes and acute myeloid leukaemia: comparison of French-AmericanBritish and World Health Organization classifications. J Hematol Oncol 2016;9:39.

107.Ok CY, Patel KP, Garcia-Manero G, et al. TP53 mutation characteristics in therapy-related myelodysplastic syndromes and acute myeloid leukemia is similar to de novo diseases. J Hematol Oncol 2015;8:45. 